Images dans le monde ibérique et ibéricoaméricain

$8 \mid 2015$

L'Histoire et ses récits entre images, fictions et paratextes

\title{
Le siège de Temixtitlán ou la bataille des mots livrée par Cortès
}

Monique Plâa

\section{(2) OpenEdition \\ Journals}

Édition électronique

URL : http://journals.openedition.org/agedor/362

DOI : 10.4000 /agedor.362

ISSN : 2104-3353

Éditeur

Laboratoire LISAA

Référence électronique

Monique Plâa, "Le siège de Temixtitlán ou la bataille des mots livrée par Cortès ", L'Âge d'or [En ligne],

8 | 2015, mis en ligne le 01 février 2016, consulté le 30 avril 2019. URL : http://

journals.openedition.org/agedor/362 ; DOI : 10.4000/agedor.362 
Monique PLÂA

Université Paris-Est Marne-la-Vallée, LISAA, EA4120

\section{Le siège de Temixtitlán ou la bataille des mots livrée par Cortès}

Résumé : La communication interrogera le surprenant récit du siège de Temixtitlán qui occupe la fin de la troisième lettre-rapport que Cortès envoie au roi d'Espagne pour l'informer et sans doute influencer favorablement son jugement. Or il s'avère que ce texte, étonnement lent semble s'éloigner de la fonction d'information et de séduction qui devrait être la sienne. On privilégiera l'observation de la nature littéraire du texte pour tenter d'en percevoir les enjeux et tenter de savoir contre qui a été gagnée la bataille des mots

Mots-clés : Cortès - Temixtitlán - discours - littérature

Resumen: En este trabajo interrogaremos el curioso relato del sitio de Temixtitlán. Este relato, como el conjunto de las cartas que Cortés mandó al rey de España, tendría que dar a conocer los avances de la conquista e influir en la opinión del monarca a favor del conquistador. Ahora bien, resulta que al escribir el relato del sitio, un relato asombrosamente lento, el autor parece olvidar la finalidad primera del texto que está redactando. Analizaremos los aspectos literarios del relato del sitio de Temixtitlán para interrogar sus fines e intentar saber contra quién se está librando la batalla de las palabras.

Palabras clave: Cortés - Temixtitlán - discurso - literatura.

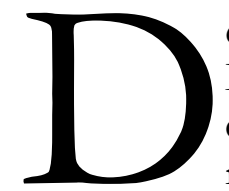

e Xénophon à Napoléon en passant par Alexandre, Annibal et Pompée, PaulMarie Duval, l'éditeur des Commentaires de César, fait une longue liste de militaires dont la plume consigne les hauts faits, mais Jules César, écrit Duval, est le seul qui rédige à chaud une œuvre qui est une marche vers le pouvoir tandis que Cortès rédige «après coup sa Relation originale pour justifier la mainmise sur le Mexique (...) » ${ }^{1}$. Or, justement, Cortès ne rédige pas ses lettres après coup. Les trois premières sont datées respectivement du 20 juillet 1519, du 30 octobre 1520 et du 15 mais 1522 et, comme le fait remarquer Ángel Delgado Gómez, éditeur du texte chez Castalia ${ }^{2}$, quand Cortès rédige sa première lettre, il ignore tout à fait ce que sera le contenu des suivantes puisqu'il écrit pendant que se déroulent les faits dont il rend compte.

Pour les lecteurs d'aujourd'hui, cette simultanéité, celle de lettres-rapports écrites pendant les faits qu'elles relatent, est moins déterminante que les conséquences terribles de ces faits : on le sait, l'effondrement de l'empire aztèque, le passage de la Conquête à la Colonie allument des polémiques sans fin. Avant même que Cortès ne parte explorer la Terre Ferme, Antonio Montesinos, dès 1511, remettait déjà en cause les modalités de la Conquête et de la Colonie et Las Casas donnera à la controverse un éclat qui résonne encore aujourd'hui. En conquérant pour l'Ancien monde, un Nouveau monde, pour l'ancienne Espagne, une Nouvelle Espagne, Cortès

${ }^{1}$ DUVAL, Paul-Marie, « Préface » in Jules César, Guerre des Gaules, Paris, Folio, 1995, p. 8.

2 DELGADO GÓMEZ, Ángel, « Introducción », in Hernán Córtés, Cartas de relación, Madrid, Castalia, 1993. 
interfère de manière décisive dans le cours de l'Histoire. Et puisque cette interférence, dès le départ et aujourd'hui encore, fait l'objet de critiques enflammées, les écrits de Cortès sont lus moins pour ce qu'ils sont que comme illustrations de savoirs ou des débats qu'on y projette a posteriori et avec de nombreux a priori.

Dans la présentation qui suit, on se propose d'inverser les priorités : alors que généralement on considère le texte de Cortès d'abord comme un document historique dont on valide ou récuse tel ou tel élément du contenu, il s'agira ici de prendre en compte la nature littéraire du texte en faisant le pari que cette nature révèle une vérité autre et peut-être plus cohérente. En effet, alors que le débat critique - et pour cause, aisément accusateur - tend à prélever telle information pour en faire un argument à charge ou à décharge, si on envisage la nature littéraire du texte, on remet la partie dans le tout et, plus que le détail, c'est la forme du récit dans son entier et dans ses divers paramètres qui guide le lecteur et sa lecture. Par ailleurs, cette démarche parait mieux adaptée pour rendre compte de la nature, à plus d'un titre étonnante, du récit du siège de Temixtitlán, la capitale du monde aztèque. Comme l'ensemble des lettres-rapports et peut-être davantage étant donné l'enjeu politique de la bataille, la fin de la troisième lettre qui contient le récit du siège doit emporter l'adhésion du roi or, précisément, elle semble peu faite pour parvenir à cette fin. On cherchera une possible explication dans la nature même du texte. Pour point de départ, on prendra l'étrangeté du récit du siège de Temixtitlán qui donne à lire une épopée qui semble aller comme à reculons. Dans un deuxième temps, c'est la nature littéraire du siège, depuis le narrateur jusqu'à la représentation de l'autre qui sera prise en compte. En conclusion, on se demandera contre qui a été gagnée la bataille des mots.

Le récit du siège de Temixtitlán est remarquable, d'abord, par l'étrange manière dont il prend fin. Dès son arrivée sur les côtes mexicaines, dès la première lettre-rapport, Cortès affirmait vouloir conquérir la tête de cet Empire dont il venait tout juste d'entendre parler mais qu'on lui disait puissant et riche. La capitale de l'empire aztèque tombe le 13 août 1521, ce qui correspond quasiment à la fin de la troisième lettre-rapport. Or, aussi étrange que cela puisse paraître, la chute de la ville est à peine mentionnée ${ }^{3}$. En effet, l'empire n'est pas plus tôt tombé que, dans le texte, se met en place une dynamique nouvelle comme l'atteste la très grande quantité de verbes d'actions qui scandent les dernières pages de la troisième lettre. Cette activité frénétique a trois orientations : Cortès fait de la ville en ruine un gigantesque chantier dont, écritil, surgira une ville plus belle encore que celle qui vient d'être détruite ; parallèlement, il lance ses ambassadeurs et ses soldats dans diverses opérations de contrôle des régions qui doivent mener à la mer du Sud soudainement devenue le nouvel horizon à conquérir ; et, pour comble, il réussit en même temps à se débarrasser d'un certain Andrés de Tapia fraîchement nommé par le roi pour occuper le poste de gouverneur de l'empire que lui, Cortès, vient de conquérir. Bref, les dernières pages de la lettre sont surprenantes parce que la chute de l'empire aztèque, que suivant la logique induite par les lettres précédentes le lecteur croyait être une fin en soi et dont on aurait pu croire que ce serait la fin tout court, n'apparait, au bout du compte, que comme une étape de la Conquête devant mener à d'autres étapes.

En revanche, si on prend la mesure du temps du récit avec les instruments de Genette, il ne fait pas de doute que les 73 jours du siège qui occupent 70 pages sont réglés par un temps lent par rapport à ce qui précède et plus lent encore par rapport à ce qui suit. La sensation de temps ralenti est d'autant plus forte que Cortès, qui pourtant rédige sa lettre quasiment deux ans après les faits, fidèle en cela au rôle de chroniqueur qui doit en principe être le sien, écrit sans tenir

\footnotetext{
3 «Aquel dia de la presión de Guatimucín y toma de la cibdad, después de haber recogido el despojo que se pudo haber nos fuemos al real, dando gracias a Nuestro Señor por tan señalada merced y tan deseada vitoria como nos había dado. Allí en el real estuve tres o cuatro días dando orden en muchas cosas que convenían, y después nos venimos a la cibdad de Coyoacán, donde hasta agora he estado entendiendo en la buena orden, gobernación, y pacificación destas partes. », CORTÉS, Hernán, Cartas de relación, Madrid, Castalia, 1993, p. 427-428. Toutes les références à cet ouvrage renvoient à cette édition.
} 
compte du dénouement de sorte que le lecteur, qui est censé lire sans savoir comment « finit l'histoire ", se trouve alors pris et empêtré dans l'égrènement d'un grand nombre de microséquences narratives qui se ressemblent et se répètent.

Il est certain que la chevauchée héroïque, « la briosa galopada de las huestes » que l'on prête parfois à Cortès ${ }^{4}$, n'apparait jamais comme telle dans son écriture : alors que l'épopée, régie par la certitude, met les moyens au service des fins, et alors que la Guerre des Gaules avance en ligne droite et fermement jusqu'à la reddition de Vercingétorix, le récit du siège de Temixtitlán, étrangement, semble comme se dérégler de l'intérieur de l'écriture qui en rend compte.

Cortès, l'homme d'action tel que le donne à lire dans ce récit l'homme de plume, est pris entre ce qu'il veut, la reddition de la ville, et ce qu'il ne veut pas, la guerre ; ce qu'il veut c'est bien prendre le contrôle de la capitale de l'empire et ce qu'il ne veut pas c'est détruire la ville et les hommes qui la défendent. Le texte, un compte-rendu méticuleux des lentes avancées suivies d'autant de replis obligés, est scandé par les ambassades que Cortès envoie à Cuauhtémoc et à ses hommes dans l'espoir de leur faire accepter la paix qu'il leur offre. Les propositions se suivent, les refus aussi. Alors que le siège se resserre, Cortès écrit :

(...) pensábamos que de cada hora se movieran a nos acometer con la paz, la cual deseábamos como a la salvación. Y ninguna cosa nos aprovechaba para los atraer a este propósito5.

Deux pages plus loin, on lit :

(...) y sin duda el día pasado y aqueste yo tenía por cierto que vinieran de paz, de la cual siempre con vitoria o sin ella hacía todas las muestras que podía, y nunca por esto en ellos hallábamos ninguna señal de paz. Y aquel día nos volvimos al real con mucho placer, aunque no nos dejaba de pesar en el alma por ver tan determinados de morir a los de la cibdad ${ }^{6}$.

Le récit du siège de Mexico est ponctué par plus d'une vingtaine de phrases de ce genre où Cortès réitère ses offres de paix de manière chaque fois plus rapprochée, chaque fois plus insistante. Et plus le temps passe, plus Cortès est en mesure d'attaquer, et plus il cherche à différer la bataille qui pourrait se solder par la ruine définitive de la ville et de ses habitants.

Ainsi donc, de manière paradoxale, Cortès, souvent contre l'avis des siens et contre l'avis de ses alliés impose une marche en avant qui va comme à reculons. Depuis la mi-juillet, les assiégés très étroitement encerclés font désormais miroiter des offres de négociation et quoiqu'elles soient suivies d'autant de faux bonds, Cortès attend encore et toujours et, prenant acte de cette étrange situation, il écrit: les Espagnols ont fait plus d'offres de paix que vaincus n'en ont jamais fait, alors que, par la grâce à Dieu, c'est nous, les Espagnols, qui sommes les vainqueurs; "aunque en nosotros vían muestras y señales de paz que jamás ningunos vencidos mostraron, siendo nosotros, con el ayuda de Nuestro Señor, los vencedores $7^{7}$. Jusqu'au bout, jusqu'au matin du 13 août 1521, jour de l'ultime attaque et de la chute de Temixtitlán, Cortès espère qu'une négociation pourra avoir lieu.

Pour expliquer les particularités de ce texte, on pourrait dire, par exemple, que si le temps est si lent c'est parce que la ville est, en effet, particulièrement difficile à prendre. On pourrait aussi supposer que si Cortès veut ménager les assiégés, c'est pour mieux reprendre leur trésor dont il avait un grand besoin ; ou imaginer que s'il veut privilégier la négociation à la destruction,

\footnotetext{
${ }^{4}$ « ¡La conquista de la Nueva España! Al fondo...un estruendo de hierro y estrépito militar revela la aspereza del cruento enfrentamiento (...) / Al contemplar la briosa galopada de la hueste de Hernán Cortés por la tierra de Nueva España (...). », ASCANIO Y TOGORES, Ramón, Teniente general del ejército, «Algunos rasgos de la personalidad militar de Hernán Cortés », Actas del primer congreso internacional sobre Hernán Cortés, Salamanca, Ediciones universidad de Salamanca, 1986, p. 18.

5 CORTÉS, Hernán, op. cit., p. 390.

${ }^{6}$ Ibid., p. 392.

${ }^{7}$ Ibid, p. 419.
} 
c'est que l'insurmontable infériorité numérique des Espagnols ne lui laisse guère le choix ; ou bien on pourrait croire que s'il répugne à détruire la ville de Temixtitlán, qui lui avait inspiré une description pleine de comparaisons hyperboliques dans sa deuxième lettre, c'est, comme il l'écrit, parce qu'elle est chère à son cœur; on pourrait surtout se risquer à penser que les questionnements qui déjà résonnent en Espagne sur le bien-fondé de la Conquête contraignent Cortès à dire son avancée le plus précautionneusement possible. Certes, sans doute toutes ces explications sont justes et recevables mais probablement très insuffisantes pour expliquer l'étrangeté d'un protagoniste qui n'avance que par force dans un récit ralenti jusqu'à l'enlisement. Il semble bien plutôt qu'il faille chercher l'explication non pas à l'extérieur mais à l'intérieur même de l'écriture, dans la manière dont le texte construit un narrateur, une action dont les personnages sont sujets ou objets. À défaut d'attester une vérité historique, le texte attestera une vérité littéraire qu'on se propose maintenant d'examiner en faisant le pari qu'elle nous en dira un peu plus sur le pourquoi de cette épopée qui va en crabe.

Parmi les clichés tenaces mais qui mériteraient d'être examinés de plus près il y a celui de Cortès manipulant le discours pour le mettre à son service. Puisque, pour une part non négligeable, le sort de son entreprise dépendait de la manière dont les lettres-rapports seraient lues et interprétées, il ne fait pas de doute que Cortès y apportait un soin tout particulier pour servir au mieux ses ambitions. Toutefois, de là à dire, par exemple, qu'il met sur le compte du « je » les actions abouties et sur celui du « nous » celles qui le sont moins, il y a, sans doute, un pas que l'examen des lettres et tout particulièrement de la partie consacrée au siège de Temixtitlán interdit que l'on franchisse. Au contraire, il apparait que, le plus souvent, Cortès tisse le « je » avec le «nous", n'hésite pas à mettre en avant ses hommes ou les actions de son bras droit, ne s'arrête jamais plus sur ses blessures que sur celles des autres et, ne perdant jamais de vue son cap, son action, Cortès tient admirablement la bride à son narcissisme. Si quelque chose frappe dans l'élaboration $\mathrm{du}$ « je » c'est, contrairement à toute attente, son extrême discrétion. Du reste, le seul revers sévère durant le siège que Cortès consigne minutieusement, celui du 30 juin, concerne justement ses hommes à lui et le met lui, Cortès ou plutôt le «je » qui figure Cortès, dans une situation d'extrême précarité de sorte que la «débâcle » est pour lui l'occasion, d'une part, de brosser un autoportrait en homme fragile et, d'autre part, de rendre un vibrant hommage aux trois hommes qui se sont relayés pour lui sauver la vie.

Bref, à la différence de Jules César, avec lequel la critique aime à le comparer, Cortès ne parle pas de lui à la troisième personne, ne crée pas un « je » pour se donner en exemple, justifier son autorité et stigmatiser les erreurs des uns ou des autres. Mais il y a plus: en effet, parallèlement à ce «je » en mode mineur, on trouve une inscription discrète mais tenace de l'attention portée à l'autre, et ce, quel que soit cet autre. Dans la réalité, pour conquérir l'empire aztèque, Cortès a dû et su gagner l'adhésion des autres, des ennemis de l'empereur aztèque, de ses sujets contraints et même de ses alliés convaincus. Pour assiéger Temixtitlán, aux côtés de Cortès il y a 900 Espagnols mais aussi 150000 Indiens sans lesquels la ville ne serait jamais tombée. Dans le texte, Cortès, qui qualifie les Indiens qui se battent à ses côtés "d'amis », ne manque jamais l'occasion de signaler qu'ils se battent « vaillamment » et quand il observe avec attention leurs rites de guerre c'est pour les admirer - par exemple lorsque les Tlaxcaltèques lancent de violents défis aux Texmixtitlèques. On ne relève dans le récit du siège ni signe de mépris ni manifestation d'une quelconque supériorité. Pourtant, la critique cite souvent un épisode brutal qui semble prouver le contraire : un matin, alors que les civils affamés tentent de trouver des aliments de survie, les Espagnols attaquent la population, femmes et enfants compris, et après l'énumération des nombreuses victimes, on lit: « (...) y así nos volvimos a nuestro real con harta presa y manjar para nuestros amigos $»^{8}$. La critique n'a pas manqué de relever le cynisme de cette remarque qui cautionne complaisamment le cannibalisme des Tlaxcaltèques. Cette caution est

\footnotetext{
${ }^{8}$ Ibid, p. 412.
} 
d'autant plus surprenante que, dans sa première lettre, Cortès écrivait à quel point la pratique du cannibalisme lui répugnait. Lors du siège de Temixtitlán, lorsque les Espagnols découvrent les leurs sacrifiés vivants au sommet de la pyramide, Cortès mentionne la tristesse que chacun en éprouve mais il n'y aucun jugement, aucune condamnation, pas même un commentaire, comme si la troisième lettre attestait une acceptation de l'autre y compris dans ses différences les plus malaisées à tolérer. Si on tient cet épisode pour une partie et qu'on le réintègre dans le tout du récit du siège, il se trouve résorbé dans l'élaboration d'un texte qui dans sa trame inscrit fortement l'attention à l'autre. On se propose maintenant d'observer brièvement les conséquences, dans le texte, de cette attention soutenue à l'autre.

On l'a précédemment signalé, le texte de Cortès n'a ni le tour ni le ton de la brillante chevauchée épique. On n'y trouvera pas non plus les formes de l'épopée telles que les fait entendre le romancero. Bernal Díaz del Castillo, qui s’amuse à insérer des romances épiques dans le récit qu'il écrit de la Conquête du Mexique, prétend que Cortès aimait réciter ce type de poème quand il n'en créait pas de sa façon. La part de jeu inhérente à toute insertion de référence identifiable par le lecteur est étrangère à l'écriture de Cortès : l'épopée n’y apparait ni avec la pompe que lui donne César dans ses Commentaires, ni avec la distance amusée que lui imprime Bernal Díaz dans sa chronique ${ }^{9}$. Sous la plume de Cortès, la rhétorique épique n'est que rarement convoquée et quand elle l'est les effets qui en résultent sont inattendus : ainsi le topique «vaincre ou mourir » est le plus souvent remplacé par un emblématique «se rendre ou mourir » ce qui a pour conséquence d'absorber l'épopée dans une tragédie entendue comme acheminement inévitable vers une destruction finale dont l'autre est le héros.

À propos du récit du siège de Mexico, Octavio Paz écrit : "También a veces parece una epopeya fantástica. El sitio de Tenochtitlán y el heroísmo de sitiadores y sitiados tiene una grandeza más épica que histórica : es Troya $»^{10}$. Mais dans le récit de Cortès, non seulement les dieux n'interfèrent pas (contrairement à une légende bien établie, Cortès ne se fait jamais passer pour le dieu de l'autre), mais de surcroît on n'y trouve non plus rien qui s'apparente à la grandiloquence héroïque inhérente aux divins héros d'Homère. De fait, dans le récit de Cortès, plus qu'à Troie, la ville qui se bat, Temixtitlán ressemble à Numance, la ville qui agonise. Dans sa Numance, Cervantès, comme Homère, convoque la déclamation, l'hyperbole, la métaphore, l'allégorie, et autres effets rhétoriques qui donnent au discours une ampleur spectaculaire propre à émouvoir et à impressionner le lecteur. Les effets épiques que l'on trouve dans le récit de Temixtitlán sont pris dans la prose comme ralentie et ils servent à dire surtout la volonté de mourir des assiégés : ils sont si rebelles, lit-on, qu’ils sont bien décidés à mourir - « (...) estaban tan rebeldes, y con la mayor muestra y determinación de morir ». Quand, vers la fin du siège, dans l'espoir d'éviter la bataille finale, Cortès envoie encore une ambassade, il s'entend répondre: «(...) que porqué así brevemente no los acababa de matar y los quitaba de penar tanto, porque ya ellos tenían deseos de morir $»^{11}$. Et lorsque le jour même de la chute de la ville, les Espagnols réussissent finalement à prendre vivant Cuauhtémoc, sous la plume de Cortès, c'est encore la mort que Cuauhtémoc demande :

Llegóse a mí y díjome en su lengua que ya él había fecho todo lo que de su parte era obligado para defenderse a sí y a los suyos fasta venir en aquel estado, que agora ficiese del lo que yo quisiese. Y puso la mano en un puñal que yo tenía, diciéndome que le diese de puñaladas y lo matase. Y yo le animé y le dije que no tuviese temor ninguno ${ }^{12}$.

\footnotetext{
${ }^{9}$ DÍAZ DEL CASTILLO, Bernal, Historia verdadera de la conquista de la Nueva España, 2 vol. Madrid, Historia 16, 1992. 10 PAZ, Octavio, «Exorcismo y liberación » in Patronato mexicano del V centenario de Cortés, A.C., Cortés, navegante, político, arquitecto, economista y literato, México, Editorial Diana, 1992, p. 18.

${ }^{11}$ CORTÉS, Hernán, op. cit., p. 419.

12 Ibid., p. 427.
} 
On peut voir dans la beauté des mots qui parent la défaite de Cuauhtémoc l'ultime déclinaison du leitmotiv de la mort qui devient plus obsédant à mesure que le récit du siège avance. La peinture épique de la fin du XVIII siècle entassera les morts aux pieds du cheval fièrement monté par le héros. L'entassement des morts sert alors à rehausser l'image du triomphateur. À la fin du récit du siège de Temixtitlán, les morts envahissent le texte et retiennent seuls l'attention du lecteur. Quand les Espagnols, désormais quasi maitres de l'ensemble de la ville, découvrent les quartiers qui leur ont résisté jusque-là, Cortès mentionne les rues jonchées de cadavres au point que pour avancer, écrit-il, on doit marcher sur les morts. Si on n'a jamais pu imaginer pareille situation, ajoute Cortès, c'est parce que les assiégés ne se débarrassaient jamais de leurs morts pour ne pas en révéler l'existence aux Espagnols et, précise-t-il, la faim et l'entassement des cadavres pestilentiels ont entrainé au moins 50000 assiégés dans la mort. Quant à ceux qui survivent encore, ils font l'objet d'une terrifiante description. Au moment de l'évacuation des civils, avant l'attaque finale, Cortès écrit : « yo había proveído que por todas las calles estuviesen españoles para estorbar que nuestros amigos no matasen a aquellos tristes que se salían, que eran sin cuento. (...) $»^{13}$. Tristes, ce seul mot, d'apparence si humble, résonne ici vraiment très fort parce que Cortès, qui fait un usage toujours mesuré du registre du sentiment, ne l'utilise que très exceptionnellement. Tristes scelle d'une note élégiaque l'agonie tragique de Temixtitlán telle que la donne à lire Cortès.

Dans la citation qui précède - et on pourrait en mentionner quantité d'autres du même genre -, on aura remarqué que Cortès écrit qu'il tente de protéger les populations assiégées de ses amis assiégeants, non pas des Espagnols, mais des Indiens et tout spécialement des Tlaxcaltèques qui, depuis le début du siège, pressent les Espagnols d'attaquer tant ils sont impatients d'abattre les Aztèques qu'ils ont en haine. On peut penser que c'est particulièrement habile de la part de Cortès de mettre la brutalité sur le compte des Indiens de son camp et les atermoiements sur le compte du « je » qui le représente dans le texte. Cependant, si on réintègre la partie dans le tout du texte, au regard de la cohérence de l'écriture, cette redoutable menace semble vraisemblable et donc vraie tant elle s'insère naturellement dans le récit de l'agonie de la ville.

Ainsi donc, on pourrait croire que c'est la forte attention portée à l'autre, à l'assiégé, qui perturbe l'épopée et l'oriente vers une forme de tragédie agonique. Et on pourrait alors voir là la cause et l'explication d'une marche en avant écrite comme une marche à reculons. Mais il reste une question essentielle. On le sait, les lettres-rapports avaient pour finalité première et urgente de convaincre leur destinataire, de le faire adhérer aux mots qui justifiaient les actes. Dans ce cadre, comment comprendre que Cortès ait rédigé et surtout expédié ce récit d'un siège où le temps s'enlise, où l'attention à l'autre ralentit l'action, où la part de l'agonie l'emporte sur celle de l'épopée ? Comment interpréter ce récit apparemment si peu fait pour séduire le roi, son destinataire ? En conclusion, on risquera donc une autre lecture du récit du siège de Temixtitlán tel que le donne à lire Cortès.

Sous plus d'un aspect, la Conquête prolonge la Reconquête. Il faut, au nom de Dieu gagner des âmes et au nom du roi, des terres et des richesses. Cortès qui, historiquement, se situe dans ce cadre-là, dépasse largement ce que ce modèle a de figé et de pragmatisme intéressé. D'ailleurs, dès que Temixtitlán tombe, on l'a dit, Cortès, jugeant sans doute que tenir n'empêche pas de courir, met aussitôt le cap sur la Mer du Sud.

Bartolomé de las Casas, qui n'est pas acteur de la Conquête mais juge de la Colonie, plaidera brillamment la cause des Indiens. Son discours, toujours exalté et flamboyant, doit sa force à l'efficacité de la vision manichéenne : les Indiens sont naturellement parés de toutes les vertus chrétiennes et gagneront le paradis puisque les Espagnols leur font souffrir le martyre ; les Espagnols, qui commettent sans discontinuer tous les péchés capitaux, sont des bourreaux, même pas des Romains, des Barbares de la pire espèce voués à l'enfer. Pour Bartolomé de las Casas,

\footnotetext{
${ }^{13}$ Ibid., p. 425.
} 
Cortès incarne l'homme impitoyable, destructeur, le loup qui dévore les tendres agneaux, l'horrible assassin auquel Neruda consacrera plus tard de si magnifiques vers. Ni Neruda ni Bartolomé de las Casas ne prêtent attention à la manière dont la part de l'autre, le sort de l'autre, la douleur de l'autre s'inscrivent dans l'écriture de Cortès, jusqu'à, apparemment, détourner de manière assez sensible le récit du siège de ce qu'on aurait pu attendre qu'il soit pour gagner la bienveillance du premier de ses destinataires.

On ne saura probablement jamais quelles forces se sont affrontées sous la plume pour que, dans l'écriture, quelque chose finisse par s'imposer contre la logique attendue du récit, contre son efficacité supposée et donc contre l'intérêt même de l'auteur. Le siège de Temixtitlán parait soumettre l'autorité de l'auteur au règne de l'écriture : à ce qu'il semble, ce sont les mots qui ont gagné la bataille.

Quand Barthes déclarait que l'auteur était mort et que "ça» parlait dans le texte, il accordait la priorité à l'écriture, à ses modalités, à ce que le texte donnait à lire. Cortès, dont le sort dépend de ses mots, moins de leur vérité que de l'écho qu'ils trouveront auprès du roi, semble avoir fait l'expérience du «ça parle ». On pourrait, en effet, croire que le récit et ce qu'il dit d'éprouvant s'est imposé, que la bride qui aurait dû tenir l'écriture s'est relâchée et que l'auteur est peut-être devenu, en effet, comme l'entendait Barthes, un premier lecteur au point de reléguer le destinataire au rôle de lecteur secondaire. En revanche, dès que le récit du siège est terminé, l'écriture redevient ce qu'elle était et tandis que le «je» du texte consigne les multiples faits et gestes sous-tendus par l'efficacité de l'action, Cortès, apparemment contre l'avis de son camp, ouvre aussitôt un chantier titanesque pour reconstruire sur les ruines fumantes de Temixtitlán une ville qu'il dit vouloir plus belle encore que la capitale qu'il vient d'abattre comme si la réalité devait se charger d'effacer les révélations de l'écriture. 